International Journal of Pure and Applied Mathematics

Volume 87 No. $6 \quad 2013,827-833$

ISSN: 1311-8080 (printed version); ISSN: 1314-3395 (on-line version)

url: http://www.ijpam.eu

doi: http://dx.doi.org/10.12732/ijpam.v87i6.11

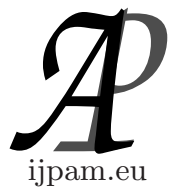

\title{
CHAIN SAMPLING PLAN FOR VARIABLE FRACTION NON-CONFORMITIES
}

\author{
K. Rebecca Jebaseeli Edna ${ }^{1}$, V. Jemmy Joyce ${ }^{2}$ \\ ${ }^{1}$ Department of Mathematics \\ Karunya University \\ Coimbatore, 641 114, India
}

\begin{abstract}
In this article a design procedure of attribute chain sampling plan for variable fraction defective is presented using stochastic differential equations. An iterative procedure for finding the parameters of the plan satisfying the given conditions with respect to producer quality level is given. Tables are constructed for easy selection of the parameters which are readily available to apply in the shaft floors of production process. The performance of the chain sampling plan for variable fraction defective is also discussed by determining the new operating characteristic function.
\end{abstract}

AMS Subject Classification: $62 \mathrm{P} 30$

Key Words: chain sampling plan, variable fraction defective, stochastic differential equations, AQL

\section{Introduction}

In Industries Acceptance Sampling Plans are widely used to control quality of finished or partly finished product. Quality control engineers use several Sampling Plans to monitor the quality of the product. These sampling plans assume that the fraction non-conformities are a constant. However in practical situation due to several uncontrolled factors the fraction non-conformities varies. Hence in this paper the fraction non- conformities is allowed to vary stochas-

Received: September 6, 2013

(c) 2013 Academic Publications, Ltd. url: www.acadpubl.eu 
tically and the system solution is obtained by solving Stochastic Differential Equations. The solution is embodied in the Acceptance Sampling Plans tool to control and monitor the product from the production process which forms as lot or batch.

A stochastic differential equation (SDE) is a differential equation in which one or more terms is a stochastic process, thus resulting in a solution which itself a stochastic process. SDE are used to model diverse phenomena such as fluctuating process parameters or physical system subject to fluctuations. Typically SDE incorporate white noise which can be thought of as the derivative of Brownian motion (or the Weiner process); however it should be mentioned that other types of random fluctuations are possible such as jump process.

Lawrence C. Evans has given an introduction to stochastic differential equations. L. Arnold(1974) has given stochastic differential equations; theory and applications. K. L. Chung(1975) has developed "Elementary probability theory with stochastic process". Schilling (1967) has given the operating procedure of attrivariate plans. Dodge H. F (1955) has developed Chain sampling inspection plans. Clark C. R (1960) has developed OC curves for Chain sampling plans. Frishman and Fred (1960) have developed an extended Chain sampling plan. Dodge. H.F \& Stephens K. S (1964) have given a general family of Chain sampling inspection plans. Dodge H. F. and Stephens K. S.(1966)have developed some new chain sampling inspection plans. Soundararajan. V (1978) has given procedure and tables for construction and selection of Chain sampling plans. Govindaraju. K and Subramani. K (1992) have designed chain sampling plans for given AQL and LQL. Govindaraju and Lai (1998) have developed modified chain sampling plans for costly or destructive items. Suresh and Devaarul (2002) have developed Mixed Sampling Plans with Chain Sampling as attribute plan.

\section{Operating Procedure of Chain Sampling Plan}

1. For each lot, select a sample of $n$ units and test each unit for conformance to the specified requirements.

2. Accept the lot if the observed number of defectives $d$ is zero, reject the lot if $d \geq 2$.

3. Accept the lot if $d$ is one and if no defective units are found in the immediately preceding $i$ samples of size $n$. 
Thus a ChSP-1 plan has two parameters namely $n$, the sample size for each submitted lot and $i$, the number of previous samples on which the decision of acceptance or rejection of the lot is based.

Dodge (1955) has given OC curves of ChSP - 1 plans with various values for $n=4,5,6 \& 10$ and $i=1,2,3,4,5 \& \infty$.

The OC function of chain sampling plan is

$$
P_{a}(p)=P_{0, n}+P_{1, n}\left(P_{0, n}\right)^{i}
$$

$P_{0, n}=$ Probability of getting exactly 0 defective in a sample of size $n$.

$P_{1, n}=$ Probability of getting exactly 1 defective in a sample of size $n$.

Hence,

$$
P_{a}(p)=e^{-n p}\left\{1+n p e^{-i n p}\right\}
$$

When $i=\infty$, the OC function of a ChSP-1 plan reduces to the OC function of single sampling plan with $c=0$. Similarly when $i=0$, the OC function of a ChSP-1 plan reduces to the OC function of single sampling plan with $c=1$.

\section{Derivation of Operating Characteristics Curve}

The OC curve of Chain Sampling Plan for variable fraction defective is derived and given below:

$$
P_{a}(p(t))=e^{-n p(t)}\left\{1+n p(t) e^{-i n p(t)}\right\}
$$

Proof. The Stochastic Differential Equation is

$$
\left\{\begin{array}{l}
\dot{X}(t)=b(X(t))+B(X(t)) \xi(t) \quad(t>0) \\
X(0)=x_{0}
\end{array}\right.
$$

where

$$
B: R^{n} \rightarrow M^{n \times m}
$$

(=space of $n \times m$ matrices) and $\xi():.=m-$ dimensional "whitenoise".

Let $P(t)$ denote the fraction non-conformities at a time $t \geq 0$. A Standard model assumes that $\frac{d P}{P}$, the relative change of defectives, evolves according to the SDE

$$
\frac{d P}{P}=\mu d t+\sigma d W
$$


In other words,

$$
\left\{\begin{array}{l}
d P=\mu P d t+\sigma P d W \\
P(0)=p_{0},
\end{array}\right.
$$

where $p_{0}$ is the initial non-conformities of the process. Using Itố $s$ formula, we have derived the model for the variable process fraction defective as

$$
P(t)=p_{0} e^{\sigma W(t)+\left(\mu-\frac{\sigma^{2}}{2}\right) t} .
$$

The OC of Chain Sampling Plan is

$$
P_{a}(p)=e^{-n p}\left\{1+n p e^{-i n p}\right\} .
$$

Suppose if the fraction defective is variable in nature then one can consider the Itố's model

$$
P(t)=p_{0} e^{\sigma W(t)+\left(\mu-\frac{\sigma^{2}}{2}\right) t} .
$$

Taking $\sigma=1, P(t)=p_{0} e^{W(t)+\frac{\mathrm{t}}{2}}$.

Hence, $P_{a}(p(t))=e^{-n p(t)}\left\{1+n p(t) e^{-i n p(t)}\right\}$.

\section{Designing and Selection of the Plan Indexed Through AQL}

1. Let the probability of acceptance of fraction defective $p_{0}$ is $1-\alpha$ and $P_{a}\left(p_{0}\right) \geq 1-\alpha$, assume $\alpha=0.01$.

2. Now determine the sample size $n$ for the given index $i$, from the following equation

$$
e^{-n p(t)}\left\{1+n p(t) e^{-i n p(t)}\right\} \geq 0.99 .
$$

3. The solution for the above equation is obtained through a computer program and given in Table 1.

Example 1. Obtain the ChSp for VFD if the known $\mathrm{AQL}=0.1 \%$ for the index $i=3$ at $99 \%$ acceptance of the process.

Solution. From table 1, the parameter of the sampling plan is $n=63$ for $i=3$.

\section{Algorithm for sentencing a lot}

Step 1: Take a random sample of size $n=63$ from a lot assumed to be large. 


\begin{tabular}{|c|c|c|c|c|c|c|c|}
\hline & Variation & & \multicolumn{5}{|c|}{ Values of $n$} \\
\cline { 4 - 8 }$p_{0}$ & $\begin{array}{c}\text { Factor } \\
\text { W }(t)-\frac{t}{2}\end{array}$ & $p_{t}$ & $i=1$ & $i=2$ & $i=3$ & $i=4$ & $i=5$ \\
\hline 0.001 & 0.1 & 0.0011 & 108 & 82 & 63 & 55 & 41 \\
\hline 0.002 & 0.2 & 0.00244 & 66 & 48 & 40 & 36 & 33 \\
\hline 0.003 & 0.3 & 0.00245 & 34 & 25 & 20 & 16 & 14 \\
\hline 0.004 & 0.4 & 0.00219 & 22 & 16 & 14 & 12 & 10 \\
\hline 0.005 & 0.5 & 0.00184 & 16 & 14 & 10 & 10 & 10 \\
\hline 0.006 & 0.6 & 0.01 & 14 & 10 & 8 & 8 & 8 \\
\hline 0.007 & 0.65 & 0.13 & 10 & 8 & 8 & 6 & 6 \\
\hline 0.008 & 0.7 & 0.16 & 8 & 8 & 6 & 6 & 5 \\
\hline 0.009 & 0.8 & 0.02 & 8 & 6 & 4 & 4 & 4 \\
\hline 0.01 & 0.9 & 0.0245 & 8 & 6 & 4 & 4 & 4 \\
\hline
\end{tabular}

Table 1: Values of $n$ for $p_{0}$ and $1-\alpha=0.99$

\begin{tabular}{|c|c|c|}
\hline$p_{0}$ & $P(t)$ & $P_{a}(p(t))$ \\
\hline 0.001 & 0.0011 & 0.9975 \\
\hline 0.002 & 0.0015 & 0.9956 \\
\hline 0.003 & 0.0022 & 0.9834 \\
\hline 0.004 & 0.0042 & 0.9298 \\
\hline 0.005 & 0.0053 & 0.9085 \\
\hline 0.01 & 0.011 & 0.6737 \\
\hline 0.03 & 0.033 & 0.4473 \\
\hline 0.05 & 0.0553 & 0.2515 \\
\hline 0.08 & 0.088 & 0.1102 \\
\hline 0.10 & 0.11 & 0.0251 \\
\hline
\end{tabular}

Table 2: OC Values: $n=66, i=1$, Variation factor $W(t)=0.1 \&$ AQL $=99 \%$

Step 2: Count the number of defectives $d$.

Step 3: If the number of defectives $d=0$, accept the lot.

Step 4: If the number of defectives is greater than 1, reject the lot.

Step 5: If the number of defectives is equal to 1 and if preceding $i=3$ samples 
Figure 1

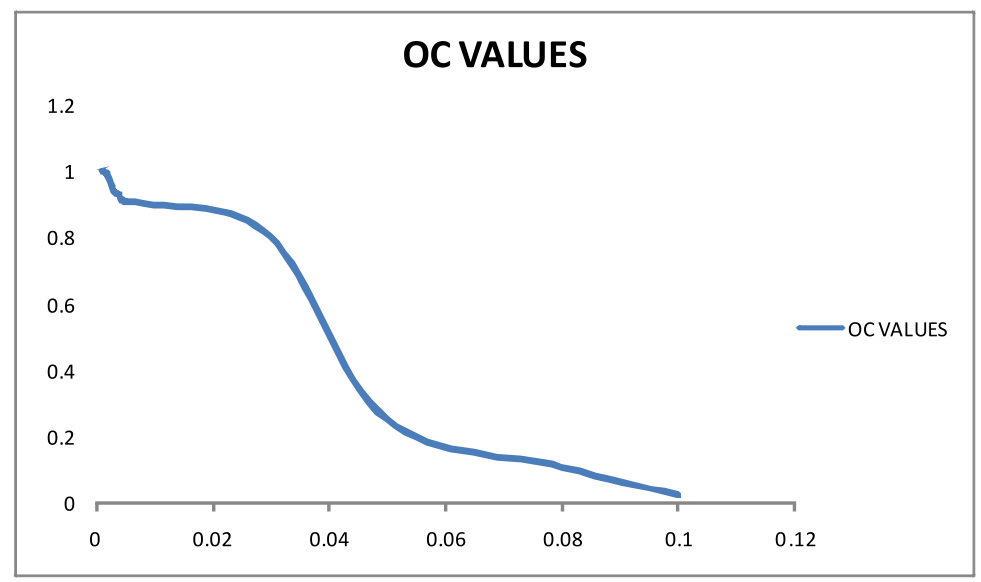

resulted in acceptance, then accept the current lot otherwise reject it.

\section{Conclusion}

The procedure outlined in this article gives the sampling plans for variable fraction non conformities. Tables are constructed to facilitate easy selection of attribute Chain Sampling Plans. The new operating characteristic curve of the plan shows the ability of the plan to distinguish between good and bad lots.

\section{References}

[1] ARNOLD. L, Stochastic Differential Equations; Theory and Applications, John Wiley \& Sons, New York (1974).

[2] CLARK. C. R, OC curves for ChSP - 1 Chain Sampling Plans, Industrial Quality Control, 17, No.4 (1960), 10-12.

[3] DODGE. H. F, Chain Sampling Inspection Plan, Industrial Quality Control, 11, No.4 (1955), 10-13.

[4] DODGE. H. F and STEPHENS. K. S, A general family of Chain sampling inspection plans, Technical Report, No.20, Rutgers - The State University, New Jersey (1964). 
[5] DODGE. H. F and STEPHENS. K. S, Some New Chain Sampling Inspection Plans, Industrial Quality Control, 23, No.2 (1966), 61-67.

[6] FRISHMAN, FRED, An Extended Chain Sampling Inspection Plan, Industrial Quality Control, 17, No.1 (1960), 10-12.

[7] GOVInDARAJU. K and LAI. C. D, A modified Chsp-1 Chain Sampling Plan, MChsp-1, with very Small Sample Sizes, American Journal of Mathematical and Management Sciences, 18, No. 3 \& 4 (1998), 343-358.

[8] GOVINDARAJU. K and SUBRAMANI. K, Selection of Chsp-1 Chain Sampling Plans for given AQL and LQL, Communications in Statistics Theory and Methods, 19, No.6 (1992), 2179-2190.

[9] LAWRANCE. C. E, An Introduction to Stochastic Differential Equations, Version 1.2, Department of Mathematics, UC Berkeley.

[10] SURESH. K. K and DEVAARUL. S, Combining Process and Product Control for Reducing Sampling Cost, Economic Quality Control, 17(2) (2002), 187-194. 
